\title{
BONUS-MALUS SYSTEMS AS MARKOV SET-CHAINS
}

BY

\author{
MaŁgorZATA NiEMieC
}

\begin{abstract}
The objective of this paper is to present an analysis of a bonus-malus system (BMS) within the framework of the theory of ergodic Markov set-chains. It is shown that this type of Markov chains enables the evaluation of BMS, even in steady-state, under the assumption that transition probabilities change in a definite range. We introduce a model that allows the determination of the consequences of changes in the claim frequency of a policyholder. In a numerical example we examine the BMS employed by one of the Polish insurance companies.
\end{abstract}

\section{KEYWORDS}

Markov set-chain, bonus-malus system, claim frequency.

\section{INTRODUCTION}

In most analyses of bonus-malus systems (BMS), it is assumed that the claim frequency of an individual policyholder remains constant. This assumption implies constant transition probabilities and makes it possible to model a BMS as a homogeneous Markov chain (see e.g. Lemaire, 1995). However, it is known that the claim frequency $\lambda$ may be time-dependant for various reasons. Moreover, actuarial tools used to evaluate BMS such as the stationary probabilities and mean first passage times are not necessarily monotonic functions of $\lambda$; describing their properties analytically is a laborious task. Therefore, it is often difficult to determine these measures' reaction to changes in claim frequency. In order to relax the assumption of a constant claim frequency, we use the concept of ergodic Markov set-chains, as defined by Hartfiel (1998).

A Markov set-chain constitutes a specific generalisation of the idea of classical Markov chains. Its fundamental assumption consists in allowing for varying transition probabilities at each step, although these changes are restricted by some lower and upper bounds. It is assumed that exact values of transition probabilities are not known: they belong to a given compact set, usually defined as an interval. 
Markov set-chains can be represented as nonhomogeneous Markov chains; however they constitute a much more efficient tool than nonhomogeneous Markov chains to study BMS. Indeed, the analysis of long-term behaviour is much easier, as the determination of all step transition matrices is not required.

This paper is organised as follows. Section 2 provides a concise theoretical description of an ergodic Markov set-chain. It is consistent with the concept developed by Hartfiel (1998), which is relatively new and has not been applied in actuarial science so far. In section 3 a model of a BMS is cast within the framework of Markov set-chain theory. In section 4 a numerical example is presented, which shows how the fluctuation of claim frequencies may affect asymptotic measures of BMS evaluation. Section 5 concludes.

\section{MARKOV SET-CHAINS}

The description of Markov set-chains presented in this section is based on Hartfiel's (1998) monograph.

\section{Definition 1.}

Let $N^{1}$ be a compact set of $r \times r$ stochastic matrices. Consider Markov chains with the state space $S=\{1,2, \ldots, r\}$, having all their transition matrices in $N^{1}$. A Markov set-chain is the sequence

$$
N^{1}, N^{2}, N^{3}, \ldots,
$$

where $N^{k}=\left\{\mathbf{P}: \mathbf{P}=\mathbf{P}_{1} \mathbf{P}_{2} \cdots \mathbf{P}_{k}\right.$, where $\mathbf{P}_{i} \in N^{1}$ for all $\left.i=1,2, \ldots, k\right\}$ for each $k$.

The set $N^{k}$ contains all possible $k$-step transition matrices, provided that one-step transition matrices belong to the set $N^{1}$. Note that $k$-step transition matrices are not uniquely determined. Since the set $N^{1}$ is compact, it is closed and bounded. In a particular case it can be an interval. We define the matrix interval as an interval

$$
[\mathbf{K}, \mathbf{Q}]=\{\mathbf{P}: \mathbf{K} \leq \mathbf{P} \leq \mathbf{Q}\},
$$

where $\mathbf{P}=\left[p_{i j}\right]$ denotes an $r \times r$ stochastic matrix and $\mathbf{K}=\left[k_{i j}\right]$ and $\mathbf{Q}=\left[q_{i j}\right]$ are nonnegative $r \times r$ matrices such that $\mathbf{K} \leq \mathbf{Q}$. As the interval $[\mathbf{K}, \mathbf{Q}]$ can be constructed by rows, it is useful to introduce also a vector interval. We define the vector interval as an interval

$$
[\mathbf{k}, \mathbf{q}]=\{\mathbf{x}: \mathbf{k} \leq \mathbf{x} \leq \mathbf{q}\},
$$

where $\mathbf{x}=\left[x_{j}\right]$ is a $1 \times r$ stochastic vector and $\mathbf{k}=\left[k_{j}\right]$ and $\mathbf{q}=\left[q_{j}\right]$ are nonnegative $1 \times r$ vectors such that $\mathbf{k} \leq \mathbf{q}$. Throughout, we assume that none of the intervals is empty. 
The important feature of the intervals described above is their tightness. A matrix interval $[\mathbf{K}, \mathbf{Q}]$ is tight if $k_{i j}=\min _{\mathbf{P} \in[\mathbf{K}, \mathbf{Q}]} p_{i j}$ and $q_{i j}=\max _{\mathbf{P} \in[\mathbf{K}, \mathbf{Q}]} p_{i j}$ for all $i$ and $j$. A vector interval $[\mathbf{k}, \mathbf{q}]$ is tight if $k_{j}=\min _{\mathbf{x} \in[\mathbf{k}, \mathbf{q}]} x_{j}$ and $q_{j}=\max _{\mathbf{x} \in[\mathbf{k}, \mathbf{q}]} x_{j}$ for all $j$. It is easy to show that for a tight vector interval the following conditions hold:

$$
k_{j}+\sum_{t \neq j} q_{t} \geq 1 \text { and } q_{j}+\sum_{t \neq j} k_{t} \leq 1 \text { for all } j .
$$

If $N^{1}$ is a tight interval [K, Q], then $\mathbf{K}$ and $\mathbf{Q}$ are column tight component bounds on $N^{1}$.

Henceforth, we restrict our analysis to Markov set-chains determined by a matrix interval $[\mathbf{K}, \mathbf{Q}]$.

Markov set-chains are classified as ergodic, regular and absorbing, in the same way as classical Markov chains. Taking into account properties of most BMS, in this paper we focus on ergodic Markov set-chains only. In order to define this type of chains, we first need to introduce the concept of an ergodic class.

The decomposition of the state space $S=\{1,2, \ldots, r\}$ of a Markov set-chain is based on the structure of the upper bound $\mathbf{Q}$ of a matrix interval $[\mathbf{K}, \mathbf{Q}]$. Through simultaneous permutations of rows and columns, $\mathbf{Q}$ can be put into the following canonical form:

$$
\mathbf{Q}=\left[\begin{array}{cccc:cccc}
\mathbf{Q}_{11} & \mathbf{0} & \cdots & \mathbf{0} & \mathbf{0} & \cdots & \mathbf{0} & \mathbf{0} \\
\mathbf{0} & \mathbf{Q}_{22} & \cdots & \mathbf{0} & \mathbf{0} & \cdots & \mathbf{0} & \mathbf{0} \\
\cdots & \cdots & \cdots & \cdots & \cdots & \cdots & \cdots & \cdots \\
\mathbf{0} & \mathbf{0} & \cdots & \mathbf{Q}_{n n} & \mathbf{0} & \cdots & \mathbf{0} & \mathbf{0} \\
\hdashline \mathbf{Q}_{n+1,1} & \mathbf{Q}_{n+1,2} & \cdots & \mathbf{Q}_{n+1, n} & \mathbf{Q}_{n+1, n+1} & \cdots & \mathbf{0} & \mathbf{0} \\
\cdots & \cdots & \cdots & \cdots & \cdots & \cdots & \cdots & \cdots \\
\mathbf{Q}_{s 1} & \mathbf{Q}_{s 2} & \cdots & \mathbf{Q}_{s n} & \mathbf{Q}_{s, n+1} & \cdots & \mathbf{Q}_{s, s-1} & \mathbf{Q}_{s s}
\end{array}\right]
$$

where $n \geq 1, \mathbf{Q}_{k k}$, is a $k \times k$ irreducible matrix for all $k=1,2, \ldots, n$, and if $t>n$ then $\mathbf{Q}_{t k} \neq \mathbf{0}$ for some $k=1,2, \ldots, t-1$. It is also assumed that all matrices from the interval $[\mathbf{K}, \mathbf{Q}]$ have undergone the same simultaneous row and column permutations. The definition of an ergodic class and an ergodic state follows.

\section{Definition 2.}

Let a Markov set-chain be determined by a matrix interval $[\mathbf{K}, \mathbf{Q}]$. Let $S_{i}$ be the class of states corresponding to $\mathbf{Q}_{i i}$ and, consequently, to the interval of submatrices $\left[\mathbf{K}_{i i}, \mathbf{Q}_{i i}\right]$. Then class $S_{i}$, where $i \leq n$, and each of its states, are called ergodic if the limit $\lim _{k \rightarrow \infty}\left[\mathbf{K}_{i i}, \mathbf{Q}_{i i}\right]^{k}$ exists and each matrix in this limit is of rank 1 . 
When determining if a class is ergodic, it is convenient to refer to the following theorem.

Theorem 1. Under the assumptions of Definition 2, the class $S_{i}$, where $i \leq n$, is ergodic if $\mathbf{K}_{i i}$ is primitive.

Now we are in a position to present the following definition.

\section{Definition 3.}

A Markov set-chain is ergodic if it has only one class and that class is ergodic.

One of the most important properties of an ergodic Markov set-chain is its convergence.

\section{Definition 4.}

The limit set of a Markov set-chain is defined as

$$
N^{\infty}=\{\mathbf{B}: \mathbf{B} \text { is a rank one matrix }\} .
$$

Theorem 2. If a Markov set-chain with a compact set of transition matrices $N^{1}$ is ergodic, then $\lim _{k \rightarrow \infty} N^{k}=N^{\infty}$.

If the set $N^{k}$ converges to the set $N^{\infty}$, and if $\mathbf{L}^{k}$ and $\mathbf{H}^{k}$ are lower and upper bounds on $N^{k}$, then the sequences $\left\{\mathbf{L}^{k}\right\},\left\{\mathbf{H}^{k}\right\}$, converge. Let their limits be denoted by $\mathbf{L}^{\infty}$ and $\mathbf{H}^{\infty}$ respectively: $\lim _{k \rightarrow \infty} \mathbf{L}^{k}=\mathbf{L}^{\infty}, \lim _{k \rightarrow \infty} \mathbf{H}^{k}=\mathbf{H}^{\infty}$. We call $\mathbf{L}^{\infty}$ and $\mathbf{H}^{\infty}$ the lower and upper limit bounds on $N^{\infty}$. Note that their rows constitute bounds on the set of stationary probability distributions.

In order to compute bounds on sets of transition matrices at each step, Hartfiel $(1991,1998)$ proposed the application of the Hi-Lo method. It is an iterative algorithm that consists in finding column tight component bounds on $N^{k}$, given the column tight component bounds on $N^{k-1}$, where $k=2,3, \ldots$ That is, column tight component bounds on $N^{1}$ produce column tight component bounds on $N^{2}$, which in turn give column tight component bounds on $N^{3}$, and so forth.

For an ergodic Markov set-chain we can also calculate bounds on mean first passage times, defined as

$$
\bar{m}_{i j}=\left[\mathbf{P}_{1}+2 \mathbf{P}_{1} \overline{\mathbf{P}}_{2}^{j}+\ldots+n \mathbf{P}_{1} \overline{\mathbf{P}}_{2}^{j} \ldots \overline{\mathbf{P}}_{n}^{j}+\ldots\right]_{i j},
$$

where $\mathbf{P}_{n} \in N^{1}$, and $\overline{\mathbf{P}}_{n}^{j}$ is the matrix obtained from $\mathbf{P}_{n}$ by replacing its $j$-th row by the row of 0 's. The matrices of lower and upper bounds on mean first passage times are denoted as $\overline{\mathbf{M}}^{l}$ and $\overline{\mathbf{M}}^{h}$ respectively. Hartfiel and Seneta (1994) proved that the sum in (2) converges and that its lower and upper bounds may be obtained via an algorithm based on the Hi-Lo method.

\section{Model of BONUS-MALUS SYSTEM}

Following Lemaire (1995), we call a system employed in automobile insurance a bonus-malus system if: 
- all policyholders of a given tariff group are divided into a finite number of classes, denoted as $C_{i}(i=1,2, \ldots, r)$, and their premium depends only on the class they belong to, and

- for each policyholder the class for a given period (usually a year) is determined uniquely by the class in the preceding period and the number of claims reported during that time.

A BMS is defined by the initial class, the premium scale, and the transition rules, which may be represented by means of $r \times r$ matrices

$$
\mathbf{T}_{k}=\left[t_{i j}^{(k)}\right] \text {, }
$$

where $t_{i j}^{(k)}=\left\{\begin{array}{lll}1 & \text { if } & T_{k}(i)=j \\ 0 & \text { if } & T_{k}(i) \neq j\end{array}\right.$ and $T_{k}(i)=j$ denotes the transfer of a policyholder reporting $k$ claims from class $C_{i}$ to class $C_{j}$ in the next period. The probability of moving from class $C_{i}$ to class $C_{j}$ for a policyholder with claim frequency $\lambda$ is given by

$$
p_{i j}(\lambda)=\sum_{k=0}^{\infty} p_{k}(\lambda) t_{i j}^{(k)}
$$

where $p_{k}(\lambda)$ is the probability that a driver with claim frequency $\lambda$ has $k$ claims in one period. Under the assumption that the claim frequency of an insured is stationary in time, a BMS can be modelled as a finite homogeneous Markov chain with the state space $S=\{1,2, \ldots, r\}$ and the transition matrix

$$
\mathbf{P}(\lambda)=\left[p_{i j}(\lambda)\right]=\sum_{k=0}^{\infty} p_{k}(\lambda) \mathrm{T}_{k} .
$$

It is worth mentioning that finite homogeneous Markov chains are irreducible and ergodic for majority of BMS in use.

The assumption of a constant claim frequency is rather unrealistic, but required for the study of BMS within the framework of homogeneous Markov chain theory. In practice, claim frequencies may change over time in response to insurance companies' actions, changes in the driving habits and behaviour of a policyholder. External factors such as weather conditions or state of roads may also play a role. Regardless of the reason for the occurrence of these changes, the need for the evaluation of their consequences is apparent. For such an analysis we should treat measures used to evaluate a BMS as functions of $\lambda$ and check their properties, particularly their monotonicity. However, for real-life BMS this is a very arduous and time-consuming task. Therefore, we propose to use ergodic Markov set-chains.

To view a BMS as a Markov set-chain we need the following assumptions:

[1] only a BMS that forms irreducible ergodic finite homogeneous Markov chain is under consideration; 
[2] the number of claims of a policyholder with claim frequency $\lambda$ conforms to a Poisson distribution;

[3] the claim frequency $\lambda$ varies in the interval $\left[\lambda^{(1)}, \lambda^{(2)}\right]$, where

$$
0<\lambda^{(1)}<\lambda^{(2)}<1 .
$$

Actual claim frequencies hardly ever exceed 1; therefore condition (3) is not restrictive in the analysis of real-life BMS. However, it is necessary that the following relationship be satisfied

$$
p_{k}(\lambda) \in\left[\min \left\{p_{k}\left(\lambda^{(1)}\right) ; p_{k}\left(\lambda^{(2)}\right)\right\}, \max \left\{p_{k}\left(\lambda^{(1)}\right) ; p_{k}\left(\lambda^{(2)}\right)\right\}\right],
$$

for $k=0,1,2, \ldots$ and $\lambda \in\left[\lambda^{(1)}, \lambda^{(2)}\right]$. It is easy to verify that $p_{0}(\lambda)$ and $p_{k}(\lambda)$ for $k=1,2, \ldots$ are respectively a decreasing and increasing function of $\lambda$ in the interval $(0,1)$, which ensures that relationship (4) holds.

Under assumptions [1]-[3] we can determine the matrix interval that comprises all transition matrices of the Markov set-chain. Lower and upper bounds on that interval can be expressed as

$$
\begin{aligned}
\mathbf{K} & =\sum_{k=0}^{\infty} \min \left\{p_{k}^{(1)}, p_{k}^{(2)}\right\} \mathbf{T}_{k}=\left[\min \left\{p_{i j}^{(1)}, p_{i j}^{(2)}\right\}\right], \\
\mathbf{Q} & =\sum_{k=0}^{\infty} \max \left\{p_{k}^{(1)}, p_{k}^{(2)}\right\} \mathbf{T}_{k}=\left[\max \left\{p_{i j}^{(1)}, p_{i j}^{(2)}\right\}\right],
\end{aligned}
$$

where superscripts ${ }^{(1)}$ and ${ }^{(2)}$ indicate that a given probability is calculated for $\lambda^{(1)}$ and $\lambda^{(2)}$, respectively. Note that $\mathbf{K}$ and $\mathbf{Q}$ are nonnegative $r \times r$ matrices such that $\mathbf{K} \leq \mathbf{Q}$. From relationship (4) and equations (5) and (6) we obtain

$$
\mathbf{P}(\lambda) \in[\mathbf{K}, \mathbf{Q}] \text { for } \lambda \in\left[\lambda^{(1)}, \lambda^{(2)}\right] .
$$

Hence, the interval $[\mathbf{K}, \mathbf{Q}]$ contains all transition matrices of irreducible, ergodic, and finite homogeneous Markov chains that describe the same BMS and differ only in the assumed value of the claim frequency of a policyholder.

Theorem 3. Let a Markov set-chain be a model of a BMS satisfying assumptions [1]-[3]. Let [K, $\mathbf{Q}$ ] be its transition matrix interval, where $\mathbf{K}$ and $\mathbf{Q}$ are given by equations (5) and (6). Then the Markov set-chain is ergodic.

PROOF: Note that the arrangement of all nonzero elements in each matrix from the interval $[\mathbf{K}, \mathbf{Q}]$ is identical and depends on the transition rules expressed in matrices $\mathbf{T}_{k}$. The interval [K, $\mathbf{Q}$ ] comprises one-step transition matrices of irreducible ergodic finite and homogeneous Markov chains. Each of these matrices is both irreducible and primitive and in its canonical form the positioning of zero and positive elements is identical. As the same pattern of zero 
and positive elements is characteristic of any matrix from the interval $[\mathbf{K}, \mathbf{Q}]$, including $\mathbf{K}$ and $\mathbf{Q}$, all matrices from this interval are irreducible and primitive. By Definition 2 and Theorem 1, the sufficient condition for the Markov setchain to be ergodic is met.

It can also be proved that the transition matrix interval $[\mathbf{K}, \mathbf{Q}]$ of the model is tight. To this end let us define two sets of indices. Let

$$
\begin{aligned}
& A_{i}=\left\{j: \min \left\{p_{i j}^{(1)}, p_{i j}^{(2)}\right\}=p_{i j}^{(1)}\right\}, \\
& B_{i}=\left\{j: \min \left\{p_{i j}^{(1)}, p_{i j}^{(2)}\right\}=p_{i j}^{(2)}\right\},
\end{aligned}
$$

where $i, j \in S$. It can be easily verified that for the above sets the conditions hold:

$$
\begin{aligned}
& A_{i}=\left\{j: \max \left\{p_{i j}^{(1)}, p_{i j}^{(2)}\right\}=p_{i j}^{(2)}\right\}, \\
& B_{i}=\left\{j: \max \left\{p_{i j}^{(1)}, p_{i j}^{(2)}\right\}=p_{i j}^{(1)}\right\} .
\end{aligned}
$$

The sets $A_{i}$ and $B_{i}$ are disjoint. Their definition and the inequality $\mathbf{K} \leq \mathbf{Q}$ imply the following relationships:

- if $t \in A_{i}$ for $i \in S$, then $p_{i t}^{(1)}$ is an element of matrix $\mathbf{K}$ and $p_{i t}^{(2)}$ is an element of matrix $\mathbf{Q}$;

- if $t \in B_{i}$ for $i \in S$, then $p_{i t}^{(2)}$ is an element of matrix $\mathbf{K}$ and $p_{i t}^{(1)}$ is an element of matrix $\mathbf{Q}$.

As an immediate consequence of the above implications we obtain

$$
\begin{aligned}
& p_{i t}^{(1)} \leq p_{i t}^{(2)} \text { for } t \in A_{i} \text { and } i, t \in S, \\
& p_{i t}^{(2)} \leq p_{i t}^{(1)} \text { for } t \in B_{i} \text { and } i, t \in S .
\end{aligned}
$$

Furthermore, referring to the stochastic property of the transition matrices $\mathbf{P}\left(\lambda^{(1)}\right)=\left[p_{i j}^{(1)}\right]$ and $\mathbf{P}\left(\lambda^{(2)}\right)=\left[p_{i j}^{(2)}\right]$ we have

$$
\sum_{t \in A_{i}} p_{i t}^{(1)}+\sum_{t \in B_{i}} p_{i t}^{(1)}=1 \text { and } \sum_{t \in A_{i}} p_{i t}^{(2)}+\sum_{t \in B_{i}} p_{i t}^{(2)}=1 \text { for } i, t \in S .
$$

Theorem 4. Let a Markov set-chain be a model of a BMS satisfying assumptions [1]-[3]. Let [K, $\mathbf{Q}$ ] be its transition matrix interval, where $\mathbf{K}$ and $\mathbf{Q}$ are given by equations (5) and (6). Then the interval $[\mathbf{K}, \mathbf{Q}]$ is tight.

Proof: Since $[\mathbf{K}, \mathbf{Q}]=\left\{\mathbf{P}: \mathbf{P}_{i} \in\left[\mathbf{K}_{i}, \mathbf{Q}_{i}\right]\right.$ for $\left.i=1,2, \ldots, r\right\}$, where $\mathbf{P}_{i}, \mathbf{K}_{i}, \mathbf{Q}_{i}$ are $i$-th rows of $\mathbf{P}, \mathbf{K}$ and $\mathbf{Q}$ respectively, it suffices to show that, for all $i \in S$, $i$-th rows of $\mathbf{K}$ and $\mathbf{Q}$ are bounds on tight vector intervals. 
If the probability $p_{i b}$ from the matrix $\mathbf{K}$ is a function of the claim frequency $\lambda^{(1)}$ then equations (7) and (9) lead to

$$
p_{i b}^{(1)}+\sum_{\substack{t \in A_{i} \\ t \neq b}} p_{i t}^{(2)}+\sum_{\substack{t \in B_{i} \\ t \neq b}} p_{i t}^{(1)} \geq p_{i b}^{(1)}+\sum_{\substack{t \in A_{i} \\ t \neq b}} p_{i t}^{(1)}+\sum_{\substack{t \in B_{i} \\ t \neq b}} p_{i t}^{(1)}=1 \text { for all } i, b, t \in S,
$$

and if $p_{i b}$ depends on $\lambda^{(2)}$ then from (8) and (9) we have

$$
p_{i b}^{(2)}+\sum_{\substack{t \in A_{i} \\ t \neq b}} p_{i t}^{(2)}+\sum_{\substack{t \in B_{i} \\ t \neq b}} p_{i t}^{(1)} \geq p_{i b}^{(2)}+\sum_{\substack{t \in A_{i} \\ t \neq b}} p_{i t}^{(2)}+\sum_{\substack{t \in B_{i} \\ t \neq b}} p_{i t}^{(2)}=1 \text { for all } i, b, t \in S .
$$

If the probability $p_{i b}$ from the matrix $\mathbf{Q}$ is a function of the claim frequency $\lambda^{(1)}$ then equations (8) and (9) imply that

$$
p_{i b}^{(1)}+\sum_{\substack{t \in A_{i} \\ t \neq b}} p_{i t}^{(1)}+\sum_{\substack{t \in B_{i} \\ t \neq b}} p_{i t}^{(2)} \leq p_{i b}^{(1)}+\sum_{\substack{t \in A_{i} \\ t \neq b}} p_{i t}^{(1)}+\sum_{\substack{t \in B_{i} \\ t \neq b}} p_{i t}^{(1)}=1 \text { for all } i, b, t \in S,
$$

and if $p_{i b}$ depends on $\lambda^{(2)}$ then from (7) and (9) we have

$$
p_{i b}^{(2)}+\sum_{\substack{t \in A_{i} \\ t \neq b}} p_{i t}^{(1)}+\sum_{\substack{t \in B_{i} \\ t \neq b}} p_{i t}^{(2)} \leq p_{i b}^{(2)}+\sum_{\substack{t \in A_{i} \\ t \neq b}} p_{i t}^{(2)}+\sum_{\substack{t \in B_{i} \\ t \neq b}} p_{i t}^{(2)}=1 \text { for all } i, b, t \in S .
$$

Thus, conditions (1) are fulfilled, which means that the vector intervals bounded by $i$-th rows of $\mathbf{K}$ and $\mathbf{Q}$ and, consequently, the matrix interval $[\mathbf{K}, \mathbf{Q}]$ are tight.

\section{Numerical EXAMPLE}

In the preceding section we have shown that Markov set-chain theory enables us to examine the consequences of claim frequency changes within a given interval. In order to illustrate the application of the model described in section 2 we analyse the BMS currently employed in first-party coverage insurance by Powszechny Zaklad Ubezpieczen SA (PZU), a Polish insurance company.

The BMS of PZU consists of 13 classes. New policyholders enter the system in class $C_{5}$. The premium levels for each class and the transition rules are given in Table 1. The properties of the system allow for modelling it both as an irreducible ergodic finite homogeneous Markov chain, and as an ergodic Markov set-chain.

Since the average claim frequency in first-party coverage insurance in Poland has been close to 0.15 over recent years, let us consider a policyholder with claim frequency varying from 0.1 to 0.2 . Having assumed that the number of claims follows the Poisson distribution, by equations (5) and (6) we get bounds $\mathbf{K}$ and $\mathbf{Q}$ on the interval comprising all possible transition matrices 
TABLE 1

BONUS-MALUS SYSTEM OF PZU

\begin{tabular}{c|c|c|c|c|c|c|c|c}
\hline \hline & & \multicolumn{7}{|c}{ Class number after } \\
\cline { 3 - 8 } $\begin{array}{c}\text { Class } \\
\text { number }\end{array}$ & $\begin{array}{c}\text { Premium level } \\
\text { (in percentage) }\end{array}$ & 0 & 1 & 2 & 3 & 4 & 5 & $\begin{array}{c}6 \text { or } \\
\text { more }\end{array}$ \\
\cline { 3 - 8 } & & \multicolumn{7}{|c|}{ claims } \\
\cline { 3 - 9 } 1 & 200 & 2 & 1 & 1 & 1 & 1 & 1 & 1 \\
2 & 150 & 3 & 1 & 1 & 1 & 1 & 1 & 1 \\
3 & 130 & 4 & 1 & 1 & 1 & 1 & 1 & 1 \\
4 & 115 & 5 & 2 & 1 & 1 & 1 & 1 & 1 \\
5 & 100 & 6 & 3 & 1 & 1 & 1 & 1 & 1 \\
6 & 90 & 7 & 4 & 2 & 1 & 1 & 1 & 1 \\
7 & 80 & 8 & 5 & 3 & 1 & 1 & 1 & 1 \\
8 & 80 & 9 & 6 & 4 & 2 & 1 & 1 & 1 \\
9 & 70 & 10 & 7 & 5 & 3 & 1 & 1 & 1 \\
10 & 60 & 11 & 8 & 6 & 4 & 2 & 1 & 1 \\
11 & 50 & 12 & 9 & 7 & 5 & 3 & 1 & 1 \\
12 & 50 & 13 & 10 & 8 & 6 & 4 & 2 & 1 \\
13 & 40 & 13 & 11 & 9 & 7 & 5 & 3 & 1 \\
\hline \hline
\end{tabular}

SourCE: General Conditions for First-Party Coverage Insurance of Powszechny Zaklad Ubezpieczen SA established on 25th April 2003.

of the ergodic Markov set-chain - the model of the BMS for the policyholder. By Theorem 4 the obtained interval is tight, and therefore the Hi-Lo method can be employed to calculate bounds on sets of each step transition matrices. Given the data we obtained lower and upper limit bounds $\mathbf{L}^{\infty}$ and $\mathbf{H}^{\infty}$, which are rank one matrices with the following rows:

$$
\mathbf{l}^{\infty}=\left[l_{j}^{\infty}\right]=\left[\begin{array}{l}
0.0000208 \\
0.0000446 \\
0.0001074 \\
0.0002213 \\
0.0005600 \\
0.0010778 \\
0.0029749 \\
0.0050562 \\
0.0162052 \\
0.0217997 \\
0.0855492 \\
0.0672202 \\
0.5140922
\end{array}\right], \mathbf{h}^{\infty}=\left[h_{j}^{\infty}\right]=\left[\begin{array}{l}
0.0024550 \\
0.0035783 \\
0.0053440 \\
0.0077067 \\
0.0117437 \\
0.0165209 \\
0.0263297 \\
0.0349721 \\
0.0615415 \\
0.0715952 \\
0.1531372 \\
0.1336289 \\
0.7789784
\end{array}\right]^{\prime}
$$


The vectors $\mathbf{l}^{\infty}$ and $\mathbf{h}^{\infty}$ are bounds on the interval of all possible stationary probability distributions. Note that their elements are minimum and maximum steady-state probabilities that the policyholder belongs to a given class, and therefore they do not add up to 1 . The variability range of the probabilities for a driver with claim frequency in the interval $[0.1,0.2]$ is diversified. Generally, in higher classes (with lower premiums) the steady-state probabilities are higher and more sensitive to the claim frequency changes. Consequently, the driver has a better chance of being in a high-discount class, however the probability of this event is subject to variations that are larger than for the probability of being in a low-discount class.

The analysis of bounds on mean first passage times may also provide valuable information. In the context of the model of a BMS each of these times indicates an average time needed by a policyholder from class $C_{i}$ to reach $C_{j}$ for the first time. In our numerical example, the matrices of lower and upper bounds on these times $\overline{\mathbf{M}}^{l}=\left[\bar{m}_{i j}^{l}\right]$ and $\overline{\mathbf{M}}^{h}=\left[\bar{m}_{i j}^{h}\right]$ are as follows:

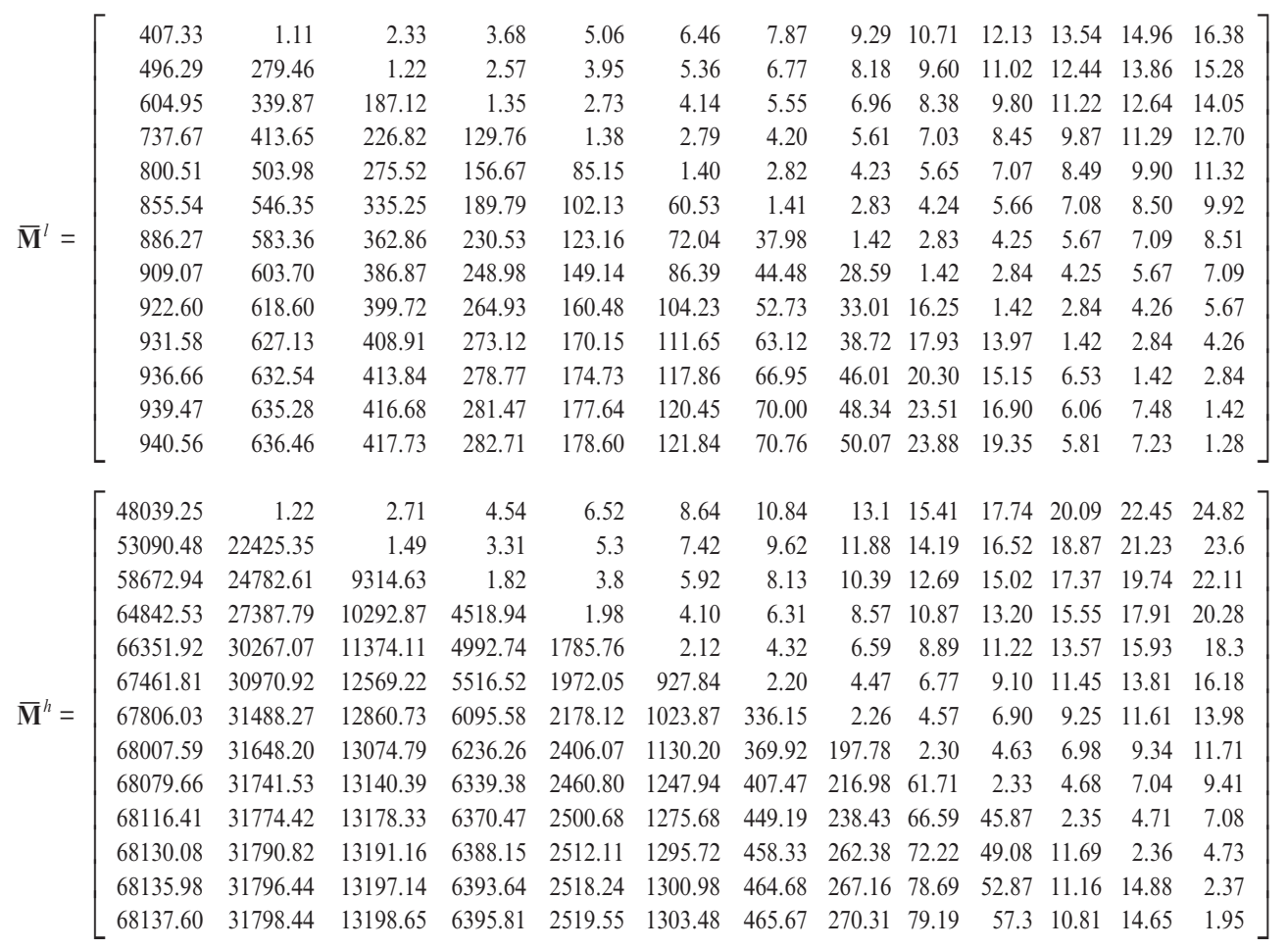

Note that the variability range of the mean first passage times is strongly diversified. It is relatively narrow for the mean times of the first promotion to higher-discount classes as well as for movement between classes $C_{9}, C_{10}, C_{11}$, $C_{12}$, and $C_{13}$. For the remaining times the range is wide, its spread exceeds 50 years and in some cases even 67000 years. Such diversity of the variability 
ranges of the mean times indicates difference in their sensitivity to the changes in claim frequency. The fluctuation of claim frequency between 0.1 and 0.2 can result in a maximal change of the mean time equal only to 0.11 (from 1.11 to 1.22 in case of the transfer from $C_{1}$ to $C_{2}$ ), as well as to over 67197 years (from 940.56 to 68137.60 in case of the transfer from $C_{13}$ to $C_{1}$ ).

The resulting values of the mean first passage times merit particular attention. For example, some transfers of a policyholder with claim frequency in the interval $[0.1,0.2]$ are virtually impossible. It is hard to expect that the policyholder will ever move to another class, if the lower bound on the mean time for such a transfer exceeds 50 years. Yet, such high values are found in approximately $40 \%$ of the elements of the matrix $\overline{\mathbf{M}}^{l}$. These are mainly lower bounds on the mean times of downgrading in the class hierarchy. This means that it is relatively difficult for the policyholder to reach a class with higher premium in the PZU system. On the other hand, in most cases the expected times for lowering a premium are significantly shorter and hence the transfer to higher-discount classes is reachable. For instance, the mean time of the first passage from the initial class $C_{5}$ to the best class $C_{13}$ amounts to 11.32 years at best, and to 18.3 years at worst, which is usually shorter than driving life. These results show that the transition rules of the system are soft for drivers with claim frequency in the interval $[0.1,0.2]$. PZU may encounter problems resulting from the clustering of policyholders in high-discount classes.

Also, it is worth mentioning that in most cases the variability ranges of stationary distribution and mean first passage times cannot be calculated by applying the theory of homogeneous Markov chains to claim frequencies 0.1 and 0.2. It follows from the fact that, in general, stationary probabilities and mean first passage times are not monotonic functions of $\lambda$. If we model the PZU system as two homogeneous Markov chains with claim frequencies 0.1 and 0.2 , we find the following stationary probability distributions:

$$
\pi^{\prime}(0.1)=\left[\pi_{j}(0.1)\right]=\left[\begin{array}{l}
0.0000208 \\
0.0000446 \\
0.0001074 \\
0.0002213 \\
0.0005601 \\
0.0010783 \\
0.0029781 \\
0.0050711 \\
0.0163053 \\
0.0221666 \\
0.0905421 \\
0.0819259 \\
0.7789784
\end{array}\right], \pi^{\prime}(0.2)=\left[\pi_{j}(0.2)\right]=\left[\begin{array}{l}
0.0024550 \\
0.0035775 \\
0.0053389 \\
0.0076864 \\
0.0116807 \\
0.0163578 \\
0.0258993 \\
0.0340366 \\
0.0590492 \\
0.0669832 \\
0.1390218 \\
0.1138214 \\
0.5140922
\end{array}\right] .
$$

Comparing the above values with the lower and upper bounds on all possible stationary probabilities obtained with the use of Markov set-chain theory, we 
observe that only for $j=1$ and $j=13$ we get $l_{j}^{\infty}=\min \left\{\pi_{j}(0.1), \pi_{j}(0.2)\right\}$ and $h_{j}^{\infty}=$ $\max \left\{\pi_{j}(0.1), \pi_{j}(0.2)\right\}$. These results suggest that the steady-state probabilities $\pi_{1}(\lambda)$ and $\pi_{13}(\lambda)$ can be monotonic functions of $\lambda$ in the interval $[0.1,0.2]$. For the remaining stationary probabilities we have $l_{j}^{\infty}<\min \left\{\pi_{j}(0.1), \pi_{j}(0.2)\right\}$ or $h_{j}^{\infty}>\max \left\{\pi_{j}(0.1), \pi_{j}(0.2)\right\}$. Similar observations relate to the mean first passage times. Applying the theory of homogeneous Markov chains we obtain the following matrices of mean first passage times $\overline{\mathbf{M}}(0.1)=\left[\bar{m}_{i j}(0.1)\right]$ and $\overline{\mathbf{M}}(0.2)=$ $\left[\bar{m}_{i j}(0.2)\right]$ for $\lambda=0.1$ and $\lambda=0.2$ respectively.

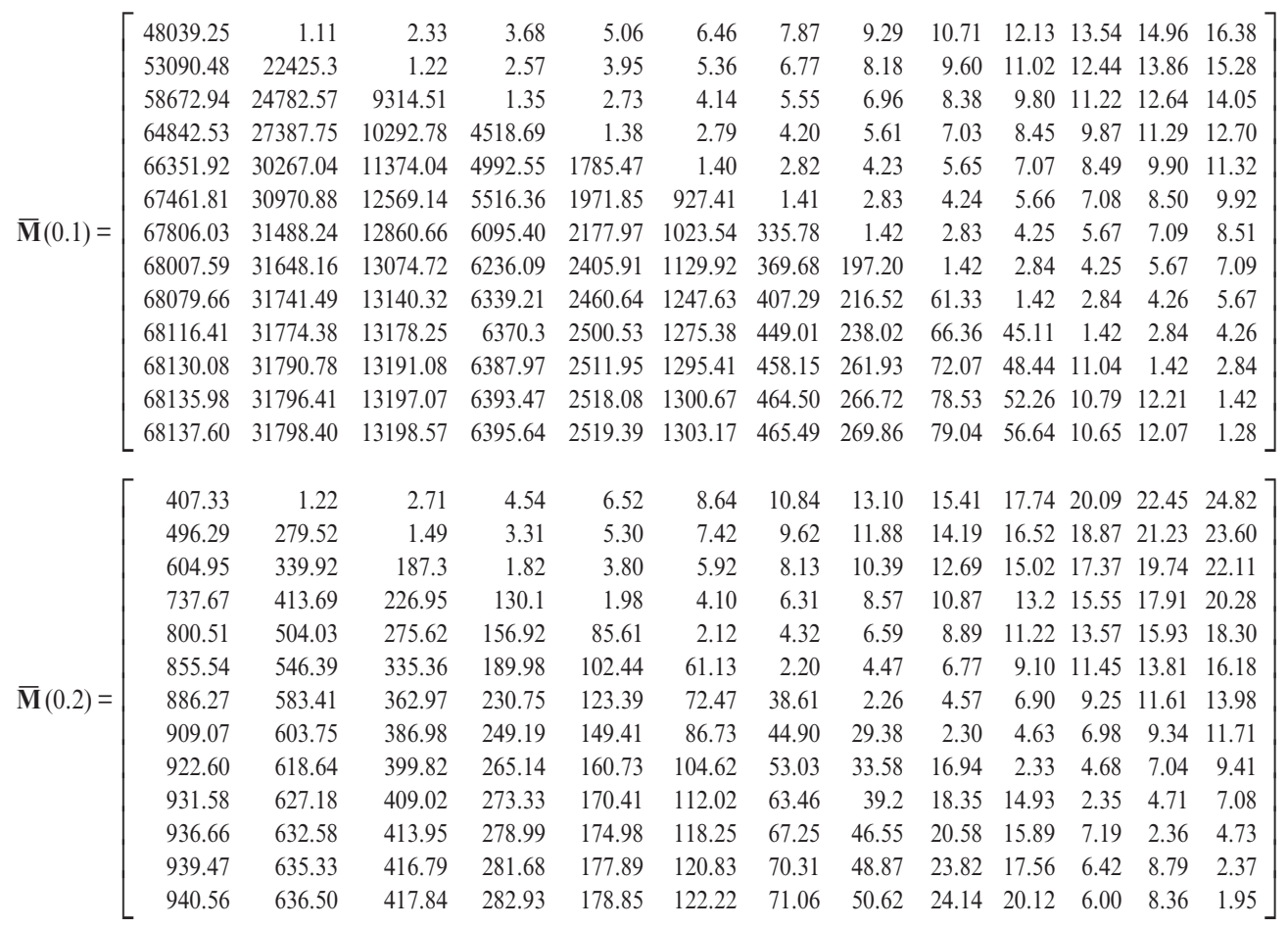

For the mean times of the first promotion to higher-discount classes and of the transfer to the worst class $C_{1}$ we have $\bar{m}_{i j}^{l}=\min \left\{\bar{m}_{i j}(0.1), \bar{m}_{i j}(0.2)\right\}$ and $\bar{m}_{i j}^{h}=$ $\max \left\{\bar{m}_{i j}(0.1), \bar{m}_{i j}(0.2)\right\}$, which may imply that these times are monotonic functions of $\lambda$ in the interval $[0.1,0.2]$. The remaining times may be neither nondecreasing nor non-increasing functions of claim frequency, as $\bar{m}_{i j}^{l}<\min \left\{\bar{m}_{i j}(0.1)\right.$, $\left.\bar{m}_{i j}(0.2)\right\}$ or $\bar{m}_{i j}^{h}>\max \left\{\bar{m}_{i j}(0.1), \bar{m}_{i j}(0.2)\right\}$. This means that most times of downgrading in the class hierarchy may increase as claim frequency grows.

\section{CONCLUSIONS}

In this paper we propose the application of ergodic Markov set-chains to the analysis of a BMS. We relax the assumption of a constant claim frequency of 
a policyholder, which is necessary when modelling the system as a homogeneous Markov chain. It is shown that the theory of the Markov set-chains broadens the scope of studies set in the framework of the classical Markov chain theory. It enables us to examine the consequences of claim frequency changes within a given interval. Moreover, it provides tools for determining the variability range of transition probabilities, stationary distributions, as well as mean first passage times. Therefore, one can analyse the sensitivity and intensity of various measures in response to the changes in claim frequency. The obtained information may be crucial to insurance companies having interest not only in BMS evaluation, but also in determining the consequences of changes in claim frequency, which they can affect to a limited extent only.

\section{ACKNOWLEDGMENT}

The author would like to thank Professor Jean Lemaire for invaluable help and detailed suggestions, which considerably improved this paper. The author is grateful to the anonymous referees for their helpful comments.

\section{REFERENCES}

HARTFiel, D.J. (1991) Component bounds for Markov set-chain limiting sets. Journal of Statistical Computation and Simulation 38, 15-24.

Hartfiel, D.J. (1998) Markov Set-Chains. Springer, New York.

Hartfiel, D.J. and Seneta, E. (1994) On the Theory of Markov-Set Chains. Advances in Applied Probability 26, 947-964.

Lemaire, J. (1995) Bonus-Malus Systems in Automobile Insurance. Kluwer Academic Publishers, Boston.

Matgorzata NiemieC

Warsaw School of Economics

Institute of Econometrics

Al. Niepodległości 164

02-554 Warsaw, Poland

Tel: +48601371611

Fax: +48225648617

E-mail:malgorzata.niemiec@sgh.waw.pl 